\title{
USO DE CHUPETA E ALEITAMENTO MATERNO EXCLUSIVOa
}

\author{
Use of pacifier and exclusive breastfeeding \\ Uso del chupete y amamantamiento materno exclusivo
}

\section{RESUMO}

Estudo quantitativo cujos objetivos foram comparar o tempo de Aleitamento Materno Exclusivo (AME) entre os grupos de idade de início do uso da chupeta e verificar a presença de associação entre o uso da chupeta e a interrupção precoce do AME. A amostra foi de 362 crianças cujas mães realizaram o pré-natal na atenção básica de saúde de Maringá, 2009. Utilizaram-se a análise ANOVA não paramétrica de Kruskal-Wallis, qui-quadrado e razão de chances. Mais da metade das crianças não fazia uso de chupeta; entre as que usavam, em $26,51 \%$ dos casos, ela foi introduzida no primeiro mês. Não foi encontrada diferença significativa entre o tempo de AME e as faixas de idade de início do uso da chupeta; no entanto, encontrou-se significância entre o grupo de crianças que não usaram chupeta com os demais grupos $(p<0,05)$. Houve associação significativa entre o uso da chupeta e o desmame precoce $(p<0,05 ; 0 R=3,2)$.

Palavras-chave: Aleitamento materno. Chupetas. Desmame. Enfermagem.

\begin{abstract}
This is a quantitative study whose objectives were to compare the time of exclusive breastfeeding (EBF) among the age groups of early pacifier use and verify the presence of an association between pacifier use and early discontinuation of EBF. The sample was from 362 children whose mothers have had prenatal care in the Health Basic Units of Maringá, in 2009. It was used Chisquare test and odds ratio at nonparametric ANOVA KruskalWallis analysis. More than half of the children did not use a pacifier, among those who use pacifier, in $26.51 \%$ of the cases, the pacifier was introduced in the first month. No significant difference was found between the duration of EBF and the age groups of early pacifier use, however significance was found between the group of children who did not use pacifiers with the other groups ( $p<0.05)$. There was a significant association between pacifier use and early weaning $(p<0.05,0 R=3.2)$.
\end{abstract}

Keywords: Breast Feeding. Pacifiers. Weaning. Nursing.

\section{Resumen}

Estudio cuantitativo cuyos objetivos fueron comparar el tiempo del Amamantamiento Materna Exclusivo (AME) entre los grupos etarios de uso temprano del chupete y verificar la presencia de una asociación entre el uso del chupete y la interrupción temprana del AME. La muestra fue realizada con 362 niños, cuyas madres recibieron atención prenatal en las Unidades Básicas de Salud de Maringá, en 2009. Se utilizó la prueba no paramétrica ANOVA de Kruskal-Wallis, la relación de Qui-Quadrado y las probabilidades. Más de la mitad de los niños no usaban chupete, entre los que usan, el $26,51 \%$ de los casos, el mismo fue introducido en el primer mes de vida. No se encontró diferencia significativa entre la duración del AME y de los grupos etarios de uso temprano del chupete, sin embargo, se encontró significación entre el grupo de niños que no usaban chupete con los otros grupos $(p<0,05)$. Existe una asociación significativa entre el uso del chupete y el destete precoz $(p<0,05,0 R=3,2)$.

Palabras-clave: Lactancia Materna. Chupetes. Destete. Enfermería.

\footnotetext{
${ }^{1}$ Enfermeira. Mestre em Enfermagem pela Universidade Estadual de Maringá (UEM)/Brasil. Professora de enfermagem da Faculdade Integrado de Campo Mourão, PR. Maringá-PR. Brasil. E-mail: mar_demitto@hotmail.com, ${ }^{2}$ Enfermeira. Doutora em Ciências Ambientais. Professora do Programa de PósGraduação em Enfermagem da UEM. Maringá - PR. Brasil. E-mail: lobercini@uem.br; ${ }^{3}$ Matemático. Doutor em Zootecnia. Professor TIDE do Departamento de Estatística da UEM. Maringá - PR. Brasil. E-mail: rmrossi@uem.br.
} 


\section{INTRODUÇÃO}

Bicos e chupetas são amplamente utilizados entre crianças de diversos países, apesar de a sua prática ter sido desaconselhada pela Organização Mundial de Saúde (OMS) ${ }^{1}$, especialmente para crianças que são amamentadas. 0 Ministério da Saúde (MS) considera seu uso danoso à saúde da criança, já que possui relação negativa com o processo de amamentação, podendo levar ao desmame; além disso, está associado à transmissão de infecções, como candidíase oral, a danos na função motora oral, exercendo papel importante na síndrome do respirador bucal, e também a problemas ortodônticos provocados pela sucção do bico?

No Brasil, a Agência Nacional de Vigilância Sanitária (ANVISA) incluiu nas embalagens dos produtos a inscrição "O Ministério da Saúde adverte: a criança que mama no peito não necessita de mamadeira, bico ou chupeta. 0 uso da mamadeira, bico ou chupeta prejudica a amamentação e seu uso prolongado prejudica a dentição e a fala da criança", a fim de restringir o uso de bicos artificiais na população ${ }^{3}$.

A OMS recomenda que as crianças sejam amamentadas exclusivamente ao seio até o sexto mês de vida e que após essa idade sejam introduzidos outros alimentos à dieta infantil, mantendo-se o aleitamento materno (AM) até os dois anos ou mais $^{2}$.

A associação entre o uso de chupeta e maior risco de interrupção do aleitamento materno exclusivo (AME) tem sido apontada em vários estudos ${ }^{4-6}$. Além disso, pesquisas que avaliaram o efeito do uso precoce da chupeta (antes de um mês de vida) sobre os desfechos posteriores da amamentação apoiam o direcionamento de causalidade, ou seja, a interrupção da amamentação pode estar sendo causada pelo ato de sucção da chupeta $^{7-8}$.

Assim, diante da necessidade de incentivo à prática de aleitamento materno, em especial do AME até os seis meses de idade e também da prevenção à introdução precoce da chupeta, considerando os malefícios que esta prática provoca na saúde da criança, este estudo tem como objetivos comparar o tempo de AME entre os grupos de idade de início do uso da chupeta, bem como verificar a presença de associação entre o uso da chupeta e a interrupção precoce do AME de crianças cujas mães realizaram o pré-natal na atenção básica de saúde do município de Maringá, no ano de 2009.

\section{MATERIAIS E MÉTODOS}

Trata-se de um estudo descritivo, exploratório, com abordagem quantitativa, realizado no município de Maringá, situado na região noroeste do Paraná, localizado a $434 \mathrm{~km}$ de Curitiba, com população de 357.117 habitantes em 2010.

Este estudo faz parte de uma pesquisa maior desenvolvida com as usuárias da rede pública de saúde que realizaram a assistência pré-natal em uma das 25 unidades básicas de saúde (UBS) do município e que foram cadastradas no Sistema de Acompanhamento do Programa de Humanização no Pré-Natal e Nascimento (SisPreNatal) até o quarto mês de gestação no ano de 2009.

Em 2009, foram atendidas nas UBS do município 1.756 gestantes segundo os critérios do SisPreNatal de consultas e exames. Por meio do Programa Epi Info versão 3.4 foi definido o tamanho amostral máximo de 315 mulheres, com 95\% de confiança e erro de estimativa de 5\%; no entanto, levando-se em consideração as eventuais perdas, o tamanho amostral ficou estabelecido em 378 mulheres (acréscimo de 20\%). A partir deste número foi realizada uma estratificação proporcional por UBS garantindo a representatividade da população amostral.

A listagem obtida no SisPreNatal, contendo o número de cadastro da gestante por UBS, foi enumerada, e a seleção das participantes do estudo foi realizada pelo cálculo dos números aleatórios no Programa Statistica $8.0^{\circledR}$, obtendose assim uma lista com a amostra representativa de gestantes por UBS.

De posse desta lista, o nome, o endereço e 0 telefone das gestantes foram obtidos no setor do SisPreNatal da Secretaria de Saúde de Maringá, por meio do programa de computador Sistema Gestor Saúde, que contém o registro de todas as mulheres que realizaram o pré-natal na rede pública de saúde do município.

Foram excluídas do estudo as gestantes que: foram a óbito; sofreram aborto; não concluíram o prénatal na rede pública de saúde do município; além das mulheres cujos bebês foram a óbito; as com condições de saúde que contraindicaram a amamentação e aquelas que se recusaram participar do estudo.

Quando uma usuária sorteada, após o contato para participação no estudo, se enquadrava em algum desses critérios, era excluída, sendo substituída pela próxima da lista.

Os dados foram coletados entre os meses de outubro de 2010 a março de 2011, por meio de entrevistas, as quais foram realizadas preferencialmente por telefone. No entanto, a dificuldade de contato telefônico com a mãe, devido a número inexistente ou não atendimento da chamada, mesmo após três tentativas em horários diferentes, fez com que 78 entrevistas fossem realizadas por meio de visita domiciliar.

Nas entrevistas foi utilizado um instrumento estruturado, constituído pelas seguintes variáveis: idade gestacional, peso ao nascer, idade da criança na entrevista, tempo de AME, uso de chupeta, idade de início e quem orientou tal prática.

Foram entrevistadas 378 mães; contudo, o total de crianças foi de 383 , visto que três mulheres tiveram gemelares e uma trigemelar. No entanto, para a presente pesquisa, a amostra foi constituída de 362 crianças, uma 
vez que 21 iniciaram o uso da chupeta com mais de 180 dias e, portanto, foram excluídas.

Os dados coletados foram analisados e os resultados foram interpretados por meio do Programa Statistica 8.0 ${ }^{\circledR}$. Para comparar o tempo de AME entre os grupos de idade de início do uso da chupeta, primeiramente foram formados três grupos, a saber: G0, referindo-se às crianças que não usaram chupeta; G1, aquelas que iniciaram antes de 30 dias; e G2, as que iniciaram entre 30 e 179 dias. Foi utilizada a ANOVA não paramétrica de Kruskal-Wallis para comparar o tempo de AME entre os grupos, visto que não houve pressupostos de normalidade nos resíduos e homogeneidade de variâncias, em nível de $5 \%$ de significância.

De acordo com a literatura, caracteriza-se introdução precoce da chupeta quando esta ocorre antes dos 30 dias de vida da criança ${ }^{7-8}$, provavelmente por esse ser um período no qual a amamentação se estabelece efetivamente ${ }^{2}$.

Para verificar a existência ou não de associação entre o uso da chupeta e a interrupção precoce do AME utilizouse o teste de qui-quadrado e a razão de chances.

A pesquisa foi aprovada pela Secretaria de Saúde do município em estudo e pelo Comitê de Ética em Pesquisa da Universidade Estadual de Maringá, conforme CAAE $n^{0}$ 0176.0.093.000-10 e parecer $n^{0}$ 256/2011 e autorizada pelas mães por meio da gravação de aceite ou apresentação e assinatura do Termo de Consentimento Livre e Esclarecido (TCLE).

\section{RESULTADOS}

A idade das crianças variou de 6 a 19 meses, sendo a média de 9,5 meses. A maioria nasceu com idade gestacional igual ou maior a 37 semanas e com peso superior a 2.500 gramas, $84,07 \%$ e $92,17 \%$, respectivamente.

Mais da metade das crianças não fazia uso de chupeta $(55,25 \%)$ e entre as que usavam, em $26,51 \%$ dos casos, ela foi introduzida no primeiro mês de vida da criança (Tabela 1).

Não foi observada diferença significativa entre 0 tempo de AME e as faixas de idade de início do uso da chupeta (G1 e G2) ( $p>0,05)$; entretanto, encontrou-se significância entre o grupo de crianças que não usaram chupeta (GO) com os demais grupos ( $p<0,05)$. Assim, entre as que não usaram chupeta (GO), a mediana de AME foi de 150 dias e entre as que usaram a mediana foi de 97,5 e 105 dias, respectivamente (G1 e G2) (Tabela 2).

Observou-se ainda associação significativa entre 0 uso da chupeta e o desmame precoce $(p<0,05)$, na qual as crianças que fizeram uso da chupeta apresentaram uma chance de 3,2 vezes mais de interromperem o AME antes dos seis meses de vida que aquelas que não fizeram uso $(\mathrm{OR}=3,2)$ (Tabela 3).

Quando da indagação sobre quem orientou o uso da chupeta, observou-se que, em $86,3 \%$ dos casos, foi a própria mãe quem decidiu oferecer a chupeta à criança, no entanto, o pai, as avós, as tias e o médico também foram citados como incentivadores do seu uso.

Tabela 1. Distribuição das crianças conforme a idade de introdução da chupeta. Maringá, Paraná, 2009.

\begin{tabular}{lll}
\hline Idade de introdução da chupeta (dias) & N & \% \\
\hline Não usa & 200 & 55,25 \\
$<30$ & 96 & 26,51 \\
30 a 179 & 66 & 18,24 \\
\hline Total & 362 & 100 \\
\hline
\end{tabular}

Tabela 2. Tempo (mediano) de AME entre os grupos de idade de início do uso da chupeta. Maringá, Paraná, 2009.

\begin{tabular}{lccc}
\hline Grupos & G0 & G1 & G2 \\
\hline Tempo de AME & $150^{\mathrm{a}}$ & $97,5^{\mathrm{b}}$ & $105^{\mathrm{b}}$ \\
$\mathrm{P}_{2,5 \%}-\mathrm{P}_{97,5 \%}$ & $15-180$ & $4-180$ & $0-180$ \\
\hline
\end{tabular}

$\mathrm{*}_{\mathrm{a}, \mathrm{b}}$ Letras distintas indicam diferenças estatisticamente significantes entre grupos por meio do teste Kruskal-Wallis $(\mathrm{p}<0,05)$.

Tabela 3. Uso de chupeta e interrupção precoce do AME. Maringá, Paraná, 2009.

\begin{tabular}{llll}
\hline Uso de chupeta & & & \\
\hline Tempo de AME & Sim & Não & Total \\
$<180$ dias & 134 & 120 & 254 \\
180 dias & 28 & 80 & 108 \\
\hline Total & 162 & 200 & 362 \\
\hline
\end{tabular}




\section{DISCUSSÃO}

0 percentual obtido de uso de chupeta no presente estudo $(44,75 \%)$ foi semelhante ao encontrado em pesquisa realizada nas capitais brasileiras ${ }^{9}$, ao verificado no estudo desenvolvido no Alto do Jequitinhonha, $\mathrm{MG}^{10}$, e ao observado em Bauru, SP ${ }^{6}$, que foram de $42,6 \%, 50,3 \%$ e $54,0 \%$, respectivamente.

Verificou-se ainda que a introdução precoce da chupeta (primeiro mês de vida da criança) ocorreu de forma frequente (26,51\%); no entanto mostrou-se inferior ao encontrado em outros estudos, como o realizado em São Paulo, SP, o qual mostrou uma taxa de $53,9 \%{ }^{4}$ e em pesquisa desenvolvida em Porto Alegre, $\mathrm{RS}$, onde $87,8 \%$ das crianças iniciaram o uso da chupeta ao longo do primeiro mês de vida ${ }^{11}$.

Não foi encontrada diferença significativa entre a faixa de idade de início do uso da chupeta e o tempo de AME, diferentemente do que foi observado em pesquisa de coorte envolvendo mães e bebês do município de Feira de Santana, $\mathrm{BA}$, na qual foi demonstrado, por meio de análise multivariada, que o uso da chupeta associou-se a um risco 53\% maior de interromper o AME no primeiro mês de lactação8.

Um estudo realizado na Dinamarca buscando investigar a influência da eficiência da técnica de mamada e do uso de chupeta na duração do AME revelou associação negativa entre o uso de chupeta (avaliado por entrevista em domićlio quando as crianças tinham em torno de 16 dias) e duração do AM, independentemente da presença de técnica inadequada da mamada. Segundo os autores, técnica inadequada de mamada e uso de chupeta criam problemas diferentes, e quando associadas aumentam ainda mais o risco de desmame precoce, motivo esse que permitiu aos pesquisadores recomendar o não uso da chupeta nas primeiras semanas de vida ${ }^{12}$.

0 estabelecimento da amamentação dá-se nas primeiras semanas de vida da criança, período no qual podem surgir alguns problemas, como ingurgitamento mamário, fissura mamilar, entre outros ${ }^{2}$. Dessa forma, o primeiro mês de vida da criança constitui-se em um momento delicado e importante para a continuidade do AM, devendo-se evitar a introdução da chupeta, a qual pode prejudicar essa fase de adaptação da amamentação.

A introdução da chupeta para crianças nas primeiras semanas de vida deve ser vista como um sinal de alerta para os profissionais de saúde, no sentido de que pode estar havendo dificuldades no processo de aleitamento materno e as mães podem estar precisando de apoio e orientações adequadas ${ }^{10}$.

$\mathrm{Na}$ análise comparativa do tempo de AME entre os grupos de idade de início do uso da chupeta, revelou-se que as crianças que não usavam chupeta foram amamentadas exclusivamente por mais tempo do que as que usavam chupeta. Resultado semelhante foi verificado em um estudo de coorte realizado em Porto Alegre, RS, no qual a incidência de desmame, entre o primeiro e sexto mês, nas crianças ainda amamentadas no fim do primeiro mês, foi de
22,4\% para as crianças não usuárias de chupeta, e de 50,8\% para as usuárias $(p<0,001)^{11}$.

A associação entre 0 uso da chupeta e o desmame precoce encontrados no presente estudo também pode ser observado em uma pesquisa de meta-análise, na qual autores analisaram estudos observacionais, a maioria coortes prospectivas, sendo 12 com AME e 9 com qualquer tipo de AM, e concluíram que o uso de chupeta estava associado à diminuiç̧ão da duração do $\mathrm{AME}$ bem como de qualquer tipo de $\mathrm{AM}^{13}$.

Uma pesquisa desenvolvida em Bauru, SP, envolvendo três inquéritos transversais que integram o projeto de monitoramento de indicadores do AM, constatou que as crianças que usavam chupeta apresentaram o dobro de chance de não estarem em AME aos seis meses de vida ( razão de prevalência $=2,03$; intervalo de confiança de $95 \% 1,44-2,84)$ e que o uso de chupeta foi o único fator associado a maior chance de interrupção do AME ${ }^{6}$.

Acredita-se que o uso da chupeta implica redução do número de mamadas por dia e, como consequência, menor estimulação do complexo mamilo-aureolar e menor produção de leite, acarretando a necessidade de complementação $0^{14-15}$. No entanto, alguns autores sugerem que a chupeta não seria a causa primária do desmame, e sim um sinal de dificuldade com a amamentação, ou mesmo ansiedade e insegurança materna diante do processo alimentar da criança, ${ }^{5,10}$. Esse fato pode explicar o elevado percentual encontrado $(86,3 \%)$ de mães que ofereceram a chupeta aos seus filhos por vontade própria. Nesses casos, esclarecer as dúvidas e tentar solucionar os problemas é mais pertinente que simplesmente retirar a chupeta.

Resultado semelhante foi observado em um estudo de coorte realizado em Porto Alegre, RS, em uma população de crianças nascidas em Hospital Amigo da Criança, que revelou que, na maioria das vezes $(72,2 \%)$ a introdução da chupeta foi feita pelas mães. As avós foram responsáveis por oferecer a chupeta em $7,2 \%$ das vezes e o pai, em 2,5\% ${ }^{11}$.

Um estudo de revisão apontou que o sucesso da amamentação está na dependência do preparo técnico, desenvolvido durante o período gravídico-puerperal, das emoções da mãe e do comportamento das pessoas que a cercam. Além disso, a introdução de bicos artificiais pode reduzir o estímulo galactogênico que é a sucção e ocasionar a diminuição da lactação, instalando-se um ciclo vicioso que pode conduzir ao desmame precoce ${ }^{14}$.

Os resultados do atual estudo são de grande relevância, já que fortalecem o conhecimento sobre o tema e podem nortear o planejamento de ações locais.

Ações de saúde sobre o não uso da chupeta, assim como o apoio à amamentação, em práticas de aconselhamento, por exemplo, são estratégias importantes para esclarecer os múltiplos desfechos negativos do uso da chupeta para a saúde do lactente ${ }^{11}$.

Nesse sentido, os profissionais de saúde precisam estar preparados para orientar as mães e a população sobre tal prática, 
estando atentos aos problemas relacionados ao processo de amamentar. Compreender o significado e a forma como as mulheres vivenciam o AM, além de valorizar seus sentimentos, dúvidas e crenças, pode contribuir para o sucesso da amamentação e, consequentemente, evitar o desmame precoce $^{16}$.

A família da nutriz precisa ser inserida nas atividades de educação em saúde sobre o uso da chupeta, visto que estas exercem influência sobre o cuidado à criança, seja essa influência ora positiva, ora negativa.

Há poucas pesquisas relacionadas ao uso da chupeta e a influência familiar ${ }^{17-18}$; portanto, são necessários estudos qualitativos que explorem esse assunto, a fim de elucidar novas estratégias de inserção da família nas ações de saúde, buscando intervir mais intensamente para que o desmame precoce não ocorra em decorrência do seu uso.

\section{CONCLUSÃO}

Ainda que o presente estudo não tenha encontrado diferença significativa entre a faixa de idade de início do uso da chupeta e o tempo de AME, ficou demonstrado que as crianças que não fizeram uso de chupeta foram amamentadas exclusivamente por mais tempo do que aquelas que usaram.

Assim, esse tema merece especial atenção nas estratégias de saúde voltadas à população infantil, não somente pela associação com o desmame precoce, mas também em função de outras repercussões negativas para a saúde e desenvolvimento da criança.

Nesse sentido, os profissionais da equipe de saúde da família precisam estar preparados para realizar as orientações, desde o pré-natal, sobre os efeitos nocivos do uso da chupeta e estimular e apoiar a nutriz na prática da amamentação, auxiliando nos problemas relacionados à lactação. É importante enfatizar que tais profissionais considerem o uso da chupeta como um sinal de alerta para a interrupção do AME, bem como um indicador de que o binômio mãe-filho necessita de um acompanhamento mais intenso da prática da amamentação.

Considerando a chupeta uma prática cultural em nosso meio, a família deve estar inserida nas atividades de educação em saúde, demonstrada a sua influência na amamentação e no cuidado geral à criança.

\section{REFERÊNCIAS}

1.World Health Organization - WHO, Organização Pan-Americana da Saúde, Ministério da Saúde (Brasil). Evidências científicas dos dez passos para o sucesso no aleitamento materno. Brasília: OPAS; 2001.
2.Ministério da Saúde (Brasil). Secretaria de Atenção à Saúde. Departamento de Atenção Básica. Saúde da Criança: nutrição infantil. Aleitamento materno e alimentação complementar. Normas e manuais técnicos. Cadernos de Atenção Básica 23. Brasilia(DF): Editora MS; 2009.

3.Resolução-RDC N 221. Diário Oficial da República Federativa do Brasil, Brasília(DF), 6 de agosto de 2002: Seção 1.

4.Cotrim LC, Venancio SI, Escuder MML. Uso de chupeta e amamentação em crianças menores de quatro meses no estado de São Paulo. Rev. bras. saúde matern. infant. 2002 set./dez; 2(3): 245-52.

5.Chaves RG, Lamounier J0, Cesar CC. Fatores associados com a duração do aleitamento materno. J Pediatr (Rio J). 2007 maio/jun.; 83(3): 241-6.

6Parizoto GM, Parada CMGL, Venancio SI, Carvalhaes MABL. Tendência e determinantes do aleitamento materno exclusivo em crianças menores de 6 meses. J Pediatr (Rio J). 2009 maio/jun.; 85(3): 2018.

7.DiGirolamo AM, Grummer-Strawn LM, Fein SB. Effect of maternity-care practices on breastfeeding. Pediatrics. 2008 june;122(2): S43-9.

8.Vieira GO, Martins CC, Vieira TO, Oliveira NF, Silva LR. Fatores preditivos da interrupção do aleitamento materno exclusivo no primeiro mês de lactação. J Pediatr (Rio J). 2010 out.; 86(5); $441-4$.

9.Ministério da Saúde (Brasil). Secretaria de Atenção à Saúde. Departamento de ações programáticas e estratégicas. II Pesquisa de Prevalência de Aleitamento Materno nas Capitais Brasileiras e Distrito Federal. Brasília(DF): Editora MS; 2009.

10.Silveira FJF, Lamounier JA. Fatores associados à duração do aleitamento materno em três municípios na região do Alto Jequitinhonha, Minas Gerais, Brasil. Cad. saúde pública. 2006 jan.; 22(1): 69-77.

11.Soares MEM, Giugliani ERJ, Braun ML, Salgado ACN, Oliveira AP, Aguiar PR. Uso de chupeta e sua relação com o desmame precoce em população de crianças nascidas em Hospital Amigo da Criança. J Pediatr (Rio J). 2003 jul./ago.; 79(4): 309-16.

12.Kronborg $\mathrm{H}$, Vaeth $\mathrm{M}$. How are effective breastfeeding technique and pacifier use related to breastfeeding problems na breatfeeding durantion? Birth. 2009 mar.; 36(1): 34-42.

13.0'Connor NR, Tanabe KO, Siadaty MS, Hauck FR. Pacifiers and breastfeeding: a systematic review. Arch Pediatr Adolesc Med. 2009 abr.; 163: 378-82.

14.Gaíva MAM, Medeiros, LS. Lactação insuficiente: uma proposta de atuação do enfermeiro. Ciênc. cuid. saúde. 2006 maio/ago.; 5(2): 255-62.

15.Castilho SD, Rocha MA. Uso de chupeta: história e visão multidisciplinar. J Pediatr (Rio J). 2009; 85(6): 480-9. 
Uso de chupeta e aleitamento materno exclusivo

Demitto MO, Bercini LO, Rossi RM

16.Catafesta F, Martins M, Zagonel IPS, Venturi KK. A amamentação na transição puerperal: o desvelamento pelo método de pesquisacuidado. Esc. Anna Nery Rev. Enferm. 2009 jul./set.;13(3): 609-16.

17.Sertório SC, Silva IA. As faces simbólica e utilitária da chupeta na visão das mães. Rev. saúde pública. 2005 abr.; 39(2): 156-62.

18.França MC, Giugliani ER, de Oliveira LD, Weigert EM, do Espírito Santo $\mathrm{LC}$, Kohler CV, et al. Uso de mamadeira no primeiro mês de vida: determinantes e influência na técnica de amamentação. Rev. saúde pública. 2008 abr.; 42(4): 607-14.

\section{NOTA}

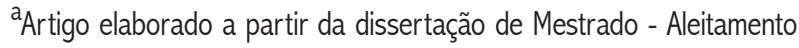
materno entre usuárias da rede pública de saúde em município da região sul do Brasil, no Curso de Mestrado em Enfermagem da Universidade Estadual de Maringá, Paraná. Subsidiado pelo Conselho Nacional de Desenvolvimento Científico-CNPq. 